nature

immunology

\title{
Embrace the complexity
}

\section{Immune cell signal transduction is just too complicated to be effectively queried using traditional methods and mindsets.}

1 ow many times has the typical immunologist been prompted-by professors, reviewers or peers-to recall what is 'upstream' or 'downstream' of an intracellular protein in the scheme of a signal emanating from a specific surface receptor? The answer is probably 'many'. Now, how many times has the typical immunologist been asked about 'lateral' inputs to that same signaling protein? The answer is probably 'far fewer'.

Implicit in the terms 'upstream' and 'downstream' is the assumption that signals in immune cells proceed in a linear pathway from receptor to nucleus. As reflected by the chuckles and gasps still heard at immunology conferences when systems biologists present slides depicting tangled knot-like schematics of immune cell signaling cascades, the reflex of many immunologists is to consider each of these pathways in isolation.

The pieces within this Focus issue (http://www.nature.com/ni/focus/ crosstalk) of Nature Immunology were specially commissioned with the intention of bolstering the concept that many influential immune cell signaling pathways actually engage in complex, often bidirectional, crosstalk with heterologous pathways. It may be more biologically meaningful to view these pathways - many of which utilize the same signaling proteins but in distinct ways — as components of larger networks, rather than as immutable, isolated tracks.

Three Review articles present the now abundant evidence for this interpathway cross-communication. Ruslan Medzhitov and Jelena Bezbradica discuss transcription-independent and transcription-dependent mechanisms through which cytokine receptor signals modulate signals emanating from heterologous receptors. They suggest that these 'lateral' interactions may explain how different cytokines, which signal through receptors sharing a very limited set of Jak and STAT signaling proteins, nevertheless manage to elicit divergent cytokine- and cell type-specific responses. Lionel Ivashkiv discusses evidence that ITAM receptors, seemingly by virtue of their capacity to activate phospholipase $\mathrm{C} \gamma$ - and calcium-dependent signaling molecules, can dampen or augment signals triggered by a wide variety of non-ITAM-containing receptors. Vishva Dixit and colleagues illustrate that the death receptor adaptors TRADD and FADD, in a manner dependent on signaling complex context and post-translational modification, facilitate apoptotic as well as non-apoptotic outcomes of death receptor signals, in addition to participating in signals emanating from several non-death receptors.

It is easy to see why this signaling interplay would make biological 'sense'. As immune cells are constantly bathed in milieus containing complex combinations of cytokines, chemokines and antigens, it is difficult to visualize a situation wherein just one pathway would be operative in any given immune cell. Adding to this complexity is the fact that migrating immune cells often move through several distinct circulatory and tissue environments. The capacity for communication between signals would allow, for example, 'tuning' of the signal emanating from one receptor in response to a change in the activity of another, and thus would help facilitate appropriate calibration of cellular responses to complex microenvironmental stimuli.

In a Perspective, John O'Shea points out another possible biological benefit of signal interplay. Although originally designed with the intention of inhibiting a single kinase, several small molecule inhibitors actually target several, often related, kinases. When used to treat tumors bearing activating mutations in a single kinase, these 'promiscuous' inhibitors have shown clinical effectiveness and less toxicity than expected. This effectiveness may stem from their capacity to inhibit several kinases in a signaling 'network'. However, another possibility is that these broad-spectrum inhibitors preemptively inhibit kinases that might be activated by subsequent mutations.

One may imagine that, although perhaps beneficial from a biological point of view, this complex signaling cross-talk would, from an experimental point of view, complicate efforts to study and understand the contribution of any one given signaling protein to immune cell behavior. In a Perspective, Victor Tybulewicz and Alexander Saveliev confirm this notion, and offer advice on how to use genetic manipulations to overcome these difficulties. Phenotypes generated using traditional 'knockout' techniques - which completely ablate expression of a signaling protein-represent the aggregate consequence of disrupting all functions of, and severing all communication facilitated by, that protein. In contrast, more refined 'knock-in' techniques—which insert a discrete mutation into only one region of a protein - allow controlled manipulation of specific protein functions and signal interconnections.

In the Overview article, Ron Germain and Iain Fraser provide further experimental advice to immunologists seeking to understand complex signaling processes that influence immune cell behavior. Urging us to adopt a 'network' rather than a 'pathway' mindset, they suggest that we need unbiased global screens conducted with stimuli in physiologically relevant amounts and ratios to identify unexpected signal cross-talk points and proteins influencing immune cell responses.

In fact, the power of these systems biology approaches is illustrated in the Research Article by Litvak et al. in this issue of Nature Immunology. Only thanks to an initial unbiased assessment of global gene expression kinetics were the authors able to identify and understand the dynamic interplay between the transcription factors NF- $\kappa \mathrm{B}, \mathrm{ATF} 3$ and $\mathrm{C} / \mathrm{EBP} \delta$. These findings are but one example of the abundant insights into immune signaling processes waiting to be harvested. Those willing to harness the tools of systems biology and shift toward a 'network' and away from a 'pathway' view of signal transduction stand the best chance of reaping this knowledge. 